\title{
Clinical heterogeneity of patients with complex sleep apnea syndrome
}

\author{
Tomasz J. Kuźniar • Kamilla Kasibowska-Kuźniar • \\ Daniel W. Ray • Thomas Freedom
}

Received: 5 November 2012 /Revised: 31 January 2013 / Accepted: 11 February 2013 /Published online: 23 February 2013

(C) The Author(s) 2013. This article is published with open access at Springerlink.com

\begin{abstract}
Background The definition of complex sleep apnea (CompSAS) encompasses patients with obstructive sleep apnea (OSA) who develop central apnea activity upon restitution of airway patency. Presence of arterial hypertension (HTN), coronary artery disease (CAD) and heart failure (HF) have been proposed as risk factors for CompSAS among OSA patients. Using our database of patients with CompSAS, we examined the prevalence of these risk factors and defined other clinical characteristics of patients with CompSAS.

Methods Through retrospective search of the database, we examined the medical and clinical characteristics of
\end{abstract}

T. J. Kuźniar • D. W. Ray

Division of Pulmonary and Critical Care Medicine, NorthShore University HealthSystem, 2650 Ridge Avenue,

Evanston, IL, USA

T. J. Kuźniar • T. Freedom

Sleep Disorders Center, NorthShore University HealthSystem, 2650 Ridge Avenue,

Evanston, IL, USA

K. Kasibowska-Kuźniar

Center of Neuropsychiatry NEUROMED,

ul. Białowieska 74A,

54-235 Wrocław, Poland

T. Freedom

Department of Neurology, NorthShore University HealthSystem, 2650 Ridge Avenue,

Evanston, IL, USA

T. J. Kuźniar $(\bowtie)$

Department of Internal Medicine, 4th Clinical Military Hospital, ul. Weigla 5 ,

53-114 Wroclaw, Poland

e-mail: tk@medycyna-snu.pl consecutive patients diagnosed with CompSAS between $11 / 1 / 2006$ and 6/30/2011 at NorthShore University HealthSystem.

Results One hundred and fifty patients with CompSAS were identified. Among patients included in the study, 97 $(64.7 \%)$ had at least one risk factor for CompSAS, while $53(35.3 \%)$ did not have any of them. Prevalence of low left ventricular ejection fraction and hypocapnia were low. Therapeutic interventions consisted of several positive airway pressure therapies, mainly adaptive servo ventilation. A hundred and ten patients $(73.3 \%)$ complied with recommended therapy and improved clinically.

Conclusions Although most patients with CompSAS have cardiac comorbidities, about one third of patients do not have any risk factors of CompSAS prior to sleep testing. Further research on factors involved in development of CompSAS will allow for better tailoring of therapy to pathophysiology involved in an individual case.

Keywords Complex sleep apnea · Comorbidities · Continuous positive airway pressure $\cdot$ Adaptive servo ventilation $\cdot$ Obstructive sleep apnea $\cdot$ Central sleep apnea

\section{Introduction}

Complex sleep apnea syndrome (CompSAS) describes the development of central apneas in a patient with obstructive sleep apnea upon treatment restoring the airway patency [1-5]. Due to its all-inclusive definition (predominance of obstructive apneas during diagnostic testing, appearance of central apneas during PAP therapy) complex sleep apnea likely represents a common phenotypic manifestation of a group of conditions. Mechanisms that are postulated in the development of CompSAS include elevated chemoreceptor 
sensitivity [6], decreased arousal threshold [7], prolonged circulation time [8], and use of opioid medications [9]. This multitude of pathways leading to CompSAS, and the improvement of some of these mechanisms with time on treatment [10] likely results in the dynamic phenotype, with the improvement or disappearance CompSAS activity in some patients [11-15], and its appearance de novo in others [14].

As a consequence of this variable etiologic background, several clinical risk factors for CompSAS among patients with OSA have been identified. CompSAS phenotype has been described in patients with congestive heart failure and Cheyne-Stokes breathing pattern [16], with up to $18 \%$ of CHF patients in a recent study exhibiting CompSAS activity [17]. The presence of arterial hypertension and coronary artery disease has been associated with CompSAS activity in other studies $[14,16]$.

It is, however, apparent from all the discussed studies and our clinical practice, that many patients with CompSAS do not have any identified "risk factors" for this condition prior to the polysomnography (PSG) evaluation. Delayed recognition of residual central sleep apnea delays institution of the effective treatment [18], and use of ineffective treatment is associated with higher risk of residual symptoms [19] and may worsen cardiovascular prognosis at least in some patient populations [20]. Using our database of CompSAS patients, we therefore aimed to estimate the prevalence of recognized risk factors among patients with CompSAS and to define other clinical and polysomnographic characteristics of these patients.

\section{Material and methods}

The study was approved by the Institutional Review Board of the NorthShore University HealthSystem. All patients were referred to a single sleep center, and evaluated between $11 / 1 / 2006$ and $06 / 30 / 2011$ by one of the team of ten sleep board-certified or board-eligible physicians. Patients underwent a diagnostic and therapeutic polysomnogram. If severe sleep apnea was observed during the diagnostic study (apnea-hypopnea index, AHI $>30 / \mathrm{h}$ ), over at least $2 \mathrm{~h}$ of recorded sleep, and sufficient time remained $(>3 \mathrm{~h}$ of expected bed time), the patient had a CPAP titration during the same night ("split-night protocol"). All studies were performed in compliance with American Academy of Sleep Medicine (AASM) standards and were scored according to the AASM scoring criteria. Airflow and respiratory effort were monitored using nasal pressure transducer and respiratory impedance plethysmography during the diagnostic study, and using the flow channel from the CPAP or BPAP plus respiratory impedance plethysmography during the positive pressure titration studies. Hypopneas were defined as an abnormal respiratory event lasting at least $10 \mathrm{~s}$ with at least a $30 \%$ reduction in thoracoabdominal movement or airflow as compared to baseline, and with at least a $4 \%$ oxygen desaturation [21].

Following the evaluation, patients were treated with PAP modalities. According to the local policies, upon the appearance of central apnea activity, bilevel PAP in the spontaneous mode (BPAP-S) was tried with expiratory PAP set at the level of CPAP that controlled obstructive events, and inspiratory PAP at the level of EPAP $+4-5 \mathrm{cmH}_{2} \mathrm{O}$. Oxygen was supplemented with PAP therapy in cases of persistent hypoxemia. In cases of ineffectiveness of BPAP-S, adaptive servo ventilation was tried, with expiratory pressure based on CPAP titration, and pressure support range left to the discretion of the physician. Final decision on therapy was made by the managing physician based on the results of all PAP studies. There was no formal policy on the use of hypnotics with PAP.

The objective compliance with the PAP device was assessed at the first visit at 4-6 weeks of its use. Patients who did not come for their follow-up appointment were contacted over the telephone regarding their usage. All patients who could not be contacted or could not supply the compliance card were treated as non-compliers.

Patients with complex sleep apnea were included in the analysis. "Complex sleep apnea" was defined as development of central sleep apnea or with central apnea index of more than 5/hour or prominent and disruptive CheyneStokes breathing pattern in a patient with obstructive sleep apnea [2]. Individual demographic and clinical variables were collected. Patients were recorded to have (1) congestive heart failure, if they had an echocardiographic documentation within 6 months of their polysomnogram of systolic or diastolic heart failure, (2) coronary artery disease, if they had a history of myocardial infarction, or a revascularization procedure, (3) hypertension, if they were on therapy for it, (4) diabetes mellitus, if they were treated for it, (5) hypothyroidism, if they were treated with thyroxin supplementation, (6) asthma or (7) chronic obstructive pulmonary disease, if they carried either diagnosis made by their primary physician and were treated, (8) stroke if they had a history of it, (9) atrial fibrillation if they had this arrhythmia in their medical history (patients did not have to be in atrial fibrillation at the time of their sleep evaluation), (10) neuromuscular weakness, (11) Parkinson's disease, or (12) cognitive impairment, if they were diagnosed and treated for those by the neurologist, (13) depression, if they were taking antidepressants at the time of sleep consultation, and (14) end-stage renal disease if they were on renal replacement therapy. Patients were noted to take opioids, benzodiazepines, or gabapentin if they were currently taking respective medications at the time of sleep consultation. 
Laboratory and auxiliary testing (pulmonary function tests, echocardiography) data were included in the analysis if they were collected within 3 months of the sleep evaluation. Serum bicarbonate level, arterial blood gases, left ventricular ejection fraction (LVEF), estimated right ventricular systolic pressure (RVSP), forced expiratory flow in one second (FEV1) and diffusing capacity of the lung for carbon monoxide (DLCO) were collected wherever available.

Continuous data were presented as medians and interquartile ranges (IQR) and analyzed using ANOVA. Discrete data were analyzed using Fisher's exact test. Correlations were analyzed with Pearson's correlation. Comparisons for serial variables were performed using a paired $t$ test.

\section{Results}

One hundred and fifty patients (117 (78 \%) males, 33 (22\%) females) with CompSAS were identified. Mean (IQR) age of the population was $62.0(53-72)$ years. At the time of sleep consultation they were found to be overweight with the body mass index (BMI) of $32.0(28.4-35.8) \mathrm{kg} / \mathrm{m}^{2}$ and had excessive daytime sleepiness with Epworth Sleepiness Scale score of $11.0(8.0-14.0)$. Significant comorbidities are presented in Table 1.

Thirty patients $(20 \%)$ had been treated for OSA prior to the evaluation. Of those, 16 were on positive airway pressure therapy (10 continuous positive airway pressure

Table 1 Comorbidities of patients with CompSAS included in the analysis. $N=150$

\begin{tabular}{ll}
\hline Comorbidity & $\begin{array}{l}\text { Number (percentage) } \\
\text { of patients }\end{array}$ \\
\hline Coronary artery disease & $37(24.7 \%)$ \\
Congestive heart failure & $32(21.3 \%)$ \\
Hypertension & $81(54.0 \%)$ \\
Stroke & $20(13.3 \%)$ \\
Atrial fibrillation & $16(10.7 \%)$ \\
Diabetes mellitus & $42(28.0 \%)$ \\
Chronic obstructive pulmonary disease & $16(10.7 \%)$ \\
Asthma & $13(8.7 \%)$ \\
Hypothyroidism & $16(10.7 \%)$ \\
Depression & $45(30.0 \%)$ \\
Neuromuscular weakness & $6(4.0 \%)$ \\
Cognitive impairment & $17(11.3 \%)$ \\
Parkinson's disease & $4(2.7 \%)$ \\
End-stage renal disease & $3(2.0 \%)$ \\
Use of medications & \\
Opioids & $28(18.7 \%)$ \\
Benzodiazepines & $6(4.0 \%)$ \\
\hline
\end{tabular}

(CPAP), 3 bilevel positive airway pressure-spontaneous (BPAP-S), 2 autoadjusting positive airway pressure (APAP), and 1 BPAP-S with oxygen), 1 was treated with uvulopalatopharyngoplasty, and 13 had been on CPAP, but had stopped this therapy prior to current evaluation.

The sleep evaluation consisted of diagnostic polysomnography (61 patients - full night, 89 - split night), with mean apnea-hypopnea index (AHI) of $50.8 \pm 29.7 / \mathrm{h}$. The results of therapeutic testing are presented in Table 2. Primary positive airway pressure testing consisted of CPAP (148 patients, $98.7 \%$ ) or BPAP-S testing (two patients, one patient with prior BPAP-S use and one patient with concurrent hypoventilation). Eventually, 70 patients $(46.7 \%)$ tried BPAP-S at some point of PAP titration, typically during a multi-modality titration study $[18,22]$. Of note, similar central apnea indices on CPAP were noted in patients studied in the full-night protocol $(21.7 \pm 21.6)$ and split-night protocol $(26.0 \pm$ 21.0, $p=0.24$ ).

Seven patients refused further evaluations and were lost to follow-up. One hundred thirteen patients underwent a titration with an adaptive servo ventilation device. Ultimately, 107 patients were prescribed an adaptive servo ventilation device, 21 patients were prescribed CPAP, 8 patients were treated with CPAP and a hypnotic, 4 patients were treated with APAP, 2 patients were treated with bilevel PAP in a spontaneous-timed mode (BPAP-ST), and one patient was treated with BPAP-S.

We then calculated the prevalence of three risk factors that have been associated with CompSAS phenotype-hypertension, coronary artery disease and congestive heart failure. Fifty-three patients $(35.3 \%)$ did not have any of the three risk factors examined, while 97 patients $(64.7 \%)$ had at least one (Fig. 1). Since one of the postulated mechanisms of CompSAS is hypocapnia leading to repeated crossing of the apneic threshold, we examined the data of 91 patients with both serum bicarbonate and LV ejection fraction. Six patients had serum bicarbonate of $<23 \mathrm{mM} / \mathrm{L}$ and 16 patients had EF of $<55 \%$ (Fig. 2).

At follow-up visit, 110 patients were using their therapy, and were significantly less sleepy than on the initial evaluation (ESS score 7.6 $\pm 5.0, p<0.001$ ). Download of the compliance device showed an average nightly use of $4.5 \pm 2.9 \mathrm{~h}$, on $70 \% \pm 40 \%$ of the nights.

\section{Discussion}

Since the first description of complex sleep apnea by Gilmartin et al [1,22], the sheer existence, definition, and treatment of this syndrome is debated [23, 24]. In our opinion, clinical utility of "CompSAS" designation lies in differences in acute and subacute response to PAP treatments of patients with "typical" OSA and CompSAS 
Table 2 Polysomnographic variables during diagnostic testing and therapeutic testing in patients with CompSAS. For CPAP and SV nights, presented data reflect "at best pressure" results. Stage- and position-dependent AHI were calculated over whole night. For adaptive servo ventilation, values represent expiratory/minimal inspiratory/ maximal inspiratory pressures

\begin{tabular}{llll}
\hline & Diagnostic study $(n=150)$ & CPAP $(n=148)$ & Adaptive servo ventilation $(n=113)$ \\
\hline Pressure & - & $11.1 \pm 3.2$ & $10.1 / 12.1 / 21.1 \pm 3.1 / 3.4 / 3.9$ \\
Apnea-hypopnea index (AHI) & $50.8 \pm 29.7$ & $39.7 \pm 29.1$ & $8.5 \pm 11.1$ \\
AHI in supine NREM sleep & $61.1 \pm 33.6$ & $37.8 \pm 23.9$ & $14.2 \pm 15.8$ \\
AHI in nonsupine NREM sleep & $34.4 \pm 36.5$ & $17.8 \pm 20.6$ & $6.4 \pm 12.3$ \\
AHI in supine REM sleep & $49.5 \pm 28.6$ & $13.4 \pm 15.7$ & $6.3 \pm 10.2$ \\
AHI in nonsupine REM sleep & $39.8 \pm 29.5$ & $11.2 \pm 16.2$ & $2.7 \pm 6.3$ \\
Central apnea index (CAI) & $6.0 \pm 8.6$ & $24.3 \pm 20.8$ & $2.6 \pm 4.8$ \\
Arousal index (AI) & $52.8 \pm 34.0$ & $36.8 \pm 23.9$ & $21.8 \pm 17.0$ \\
Periodic limb movement index (PLMI) & $26.3 \pm 39.1$ & $20.3 \pm 28.9$ & $24.0 \pm 29.3$ \\
Periodic limb movement arousal index (PLMAI) & $9.5 \pm 19.4$ & $7.9 \pm 14.0$ & $8.5 \pm 10.6$ \\
\hline
\end{tabular}

patients: while CPAP is highly effective in OSA, its acute effectiveness in much lower in CompSAS [25]. This low acute effectiveness of CPAP (documented again in our patients) translates into poorer subjective benefit of CompSAS patients than OSA patients when they are treated with CPAP [19]; such a poor early subjective benefit has been associated with poorer long term use among OSA patients [26]. Additionally, in a substantial proportion of patients with CompSAS, long-term effects of therapy with CPAP are also suboptimal $[12,27]$. Therefore, accepting the limitations of current definition of CompSAS, which is purely based on polysomnographic variables, we attempted to define the broad clinical characteristics of patients with CompSAS.

Our study represents the largest group of patients with CompSAS described clinically to date. Cassel et al. described 82 patients with CompSAS in their prospective observational study [14], while Westhof et al. described 56 patients with central apnea activity, but included only patients with normal B-type natriuretic peptide levels [15]. Finally, Javaheri et al. examined 84 patients with central apnea activity on CPAP, but included in this group those

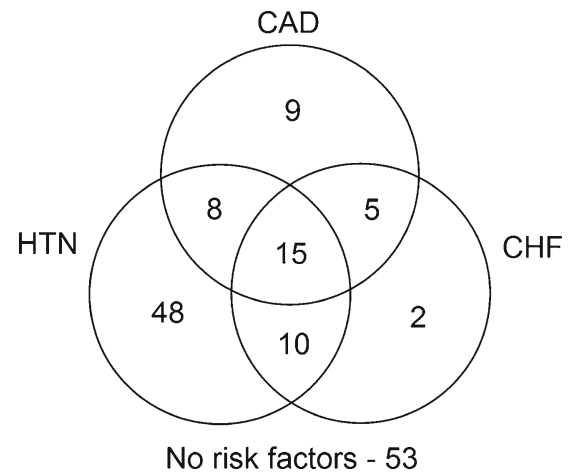

Fig. 1 Prevalence of hypertension (HTN), coronary artery disease (CAD), and congestive heart failure (CHF) among 150 patients with complex sleep apnea with central sleep apnea [13]. Hypertension was present in over half of our patients, which confirms the findings of Westhof et al. and Cassel et al., who found hypertension in 72 and $71 \%$ of their patients with CompSAS, respectively $[14,15]$. Our dataset contained significantly more patients with CompSAS and heart failure than in populations described by other authors [13-16]. Coronary artery disease, diabetes, and depression were all present in more than $20 \%$ patients of our population. Our laboratory data analysis showed changes typical of comorbidities: patients treated with opioids had hypercapnia, while patients with $\mathrm{CHF}$ tended to have lower LVEF. Though CompSAS activity has been reported in opioid users, this patient population has not been identified as a risk factor for CompSAS [28-30].

In our patient population, over one third of patients did not have any recognized risk factors for CompSAS and this diagnosis was unexpected prior to PSG testing. This suggests that different mechanisms are responsible for

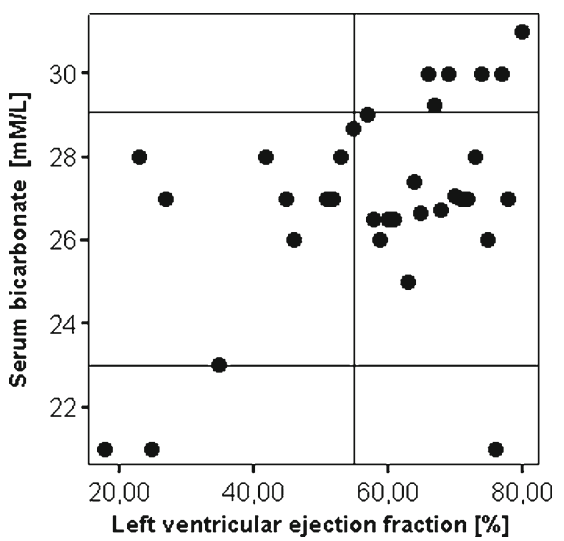

Fig. 2 Serum bicarbonate level [in millimoles per liter] and left ventricular ejection fraction [LVEF, \%] in 91 patients with CompSAS. Lines represent limits of normal values $(23-29 \mathrm{mM} / \mathrm{L}$ for serum bicarbonate and $55 \%$ for LVEF) 
Table 3 Clinical characteristics of patients with CompSAS (as compared to pts with OSA) in published series

\begin{tabular}{|c|c|c|c|c|c|c|}
\hline & $\begin{array}{l}\text { Pusalavidyasagar } \\
\text { et al [19] }\end{array}$ & Lehman et al [16] & Javaheri et al $[13]^{\mathrm{a}}$ & Endo et al [33] & Yaegashi et al [32] & Cassel et al [14] \\
\hline Age & NS & NS & - & NS & NS & $p<0.01$ \\
\hline Gender & $p<0.05$ & $p<0.05$ & - & NS & NS & NS \\
\hline BMI & $p<0.05$ & NS & NS & NS & NS & NS \\
\hline ESS & NS & - & NS & - & - & NS \\
\hline AHI & NS & $p<0.05$ & $p<0.01$ & $p<0.01$ & NS & $p<0.01$ \\
\hline CAI & $p<0.05$ & $p<0.01$ & $p<0.01$ & - & NS & $p<0.01$ \\
\hline HTN & NS & - & - & NS & NS & $p<0.05$ \\
\hline CAD & - & $p<0.05$ & NS & NS & NS & $p<0.01$ \\
\hline $\mathrm{CHF}$ & NS & $p<0.05$ & NS & NS & - & NS \\
\hline Stroke & - & NS & - & NS & NS & NS \\
\hline Atrial fibrillation & NS & - & NS & - & - & NS \\
\hline Opioids & - & NS & NS & - & - & NS \\
\hline
\end{tabular}

${ }^{a}$ Patients with CompSAS and OSA were matched for age and BMI

appearance of CompSAS phenotype in different patients; polysomnography is likely to be the one of the modalities used to establish the exact mechanism in an individual patient but it is unlikely to be the only one. Measurement of chemoresponsiveness, arousal threshold or controller gain may improve the accuracy of this diagnosis, but are still experimental and not in daily clinical use [31]. Breathto-breath variability prior to sleep onset was found to be higher in patients with mixed and central apnea tendency (and presumably higher chemoresponsiveness) that in patients with pure obstructive sleep apnea [32].

Treatment of CompSAS is another area of debate, and various PAP modalities have been used [25, 33]. Since central component of CompSAS resolves with time in some patients, CPAP has been proposed as therapy, especially in those patients who do not have cardiac or pulmonary comorbidities $[11,13]$. In our sample, adaptive servo ventilation was used most frequently. This reflects both the PAP titration policies and practice style at NorthShore University HealthSystem and is backed up by recent data showing an advantage of ASV over CPAP [27, 34].

The major limitation of the study is its retrospective design. This, specifically, may have introduced a type II error in our analyses pertaining to the physiological variables we gathered in our patients. However, given the low prevalence of CompSAS (1-15\% based on most studies) [2, 13, 14], the setup of a large prospective study systematically examining a similar scope of clinical data would be difficult. The design of our study did not allow for direct comparison of prevalence of comorbidities between CompSAS and OSA patients. In various studies reported in the literature, higher age, male gender, individuals with lower BMI, higher baseline $\mathrm{AHI}$ and $\mathrm{CAI}$, coronary artery disease, hypertension and congestive heart failure were more frequently associated with higher prevalence of CompSAS than matched patients with OSA (Table 3), with the significant differences among the studies. We speculate that these differences were due to different ethnic backgrounds of populations studied, which included Americans [2, 12, 13, 19], Australians [16], Japanese [35, 36], and European Caucasians [14] and varying referral patterns in different sleep labs. For the purpose of our analysis, patients diagnosed with CompSAS based on a split-night study were combined with patients in whom the diagnosis was established on the basis of a full-night study, which might be a potential source of bias. Given the descriptive character of this study, we do not think that this bias is significant; also, there were no statistically significant differences in all polysomnographic variables tested between patients diagnosed with CompSAS in a full-night vs split-night protocol.

In summary, we have documented the clinical heterogeneity in the largest sample of consecutive patients with complex sleep apnea examined to date. Further research in CompSAS should be focused on better delineation of the interplay of pathophysiological chains involved which will hopefully translate into more accurate prediction of CompSAS activity and better tailoring of therapy in this group of patients.

Open Access This article is distributed under the terms of the Creative Commons Attribution License which permits any use, distribution, and reproduction in any medium, provided the original author(s) and the source are credited.

\section{References}

1. Gilmartin GS, Daly RW, Thomas RJ (2005) Recognition and management of complex sleep-disordered breathing. Curr Opin Pulm Med 11:485-493 
2. Morgenthaler TI, Kagramanov V, Hanak V, Decker PA (2006) Complex sleep apnea syndrome: is it a unique clinical syndrome? Sleep 29:1203-1209

3. Kuzniar TJ, Kovacevic-Ristanovic R, Freedom T (2011) Complex sleep apnea unmasked by the use of a mandibular advancement device. Sleep Breath 15:249-252

4. Corcoran S, Mysliwiec V, Niven AS, Fallah D (2009) Development of central sleep apnea after maxillofacial surgery for obstructive sleep apnea. J Clin Sleep Med 5:151-153

5. Goldstein C, Kuzniar TJ (2012) The emergence of central sleep apnea after surgical relief of nasal obstruction in obstructive sleep apnea. J Clin Sleep Med 8:321-322

6. Younes M, Ostrowski M, Atkar R, Laprairie J, Siemens A, Hanly P (2007) Mechanisms of breathing instability in patients with obstructive sleep apnea. J Appl Physiol 103:1929-1941

7. Berry RB, Gleeson K (1997) Respiratory arousal from sleep: mechanisms and significance. Sleep 20:654-675

8. Tkacova R, Niroumand M, Lorenzi-Filho G, Bradley TD (2001) Overnight shift from obstructive to central apneas in patients with heart failure: role of $\mathrm{PCO} 2$ and circulatory delay. Circulation 103:238-243

9. Walker JM, Farney RJ, Rhondeau SM, Boyle KM, Valentine K, Cloward TV, Shilling KC (2007) Chronic opioid use is a risk factor for the development of central sleep apnea and ataxic breathing. J Clin Sleep Med 3:455-461

10. Spicuzza L, Bernardi L, Balsamo R, Ciancio N, Polosa R, Di Maria G (2006) Effect of treatment with nasal continuous positive airway pressure on ventilatory response to hypoxia and hypercapnia in patients with sleep apnea syndrome. Chest 130:774-779

11. Dernaika T, Tawk M, Nazir S, Younis W, Kinasewitz GT (2007) The significance and outcome of continuous positive airway pressure-related central sleep apnea during split-night sleep studies. Chest 132:81-87

12. Kuzniar TJ, Pusalavidyasagar S, Gay PC, Morgenthaler TI (2008) Natural course of complex sleep apnea-a retrospective study. Sleep Breath 12:135-139

13. Javaheri S, Smith J, Chung E (2009) The prevalence and natural history of complex sleep apnea. J Clin Sleep Med 5:205-211

14. Cassel W, Canisius S, Becker HF, Leistner S, Ploch T, Jerrentrup A, Vogelmeier C, Koehler U, Heitmann J (2011) A prospective polysomnographic study on the evolution of complex sleep apnoea. Eur Respir J 38:329-337

15. Westhoff M, Arzt M, Litterst P (2012) Prevalence and treatment of central sleep apnoea emerging after initiation of continuous positive airway pressure in patients with obstructive sleep apnoea without evidence of heart failure. Sleep Breath 16:71-78

16. Lehman S, Antic NA, Thompson C, Catcheside PG, Mercer J, McEvoy RD (2007) Central sleep apnea on commencement of continuous positive airway pressure in patients with a primary diagnosis of obstructive sleep apnea-hypopnea. J Clin Sleep Med 3:462-466

17. Bitter T, Westerheide N, Hossain MS, Lehmann R, Prinz C, Kleemeyer A, Horstkotte D, Oldenburg O (2011) Complex sleep apnoea in congestive heart failure. Thorax 66:402-407

18. Kuzniar TJ, Golbin JM, Morgenthaler TI (2007) Moving beyond empiric continuous positive airway pressure (CPAP) trials for central sleep apnea: a multi-modality titration study. Sleep Breath 11:259-266

19. Pusalavidyasagar SS, Olson EJ, Gay PC, Morgenthaler TI (2006) Treatment of complex sleep apnea syndrome: a retrospective comparative review. Sleep Med 7:474-479
20. Arzt M, Floras JS, Logan AG, Kimoff RJ, Series F, Morrison D, Ferguson K, Belenkie I, Pfeifer M, Fleetham J, Hanly P, Smilovitch M, Ryan C, Tomlinson G, Bradley TD (2007) Suppression of central sleep apnea by continuous positive airway pressure and transplant-free survival in heart failure: a post hoc analysis of the Canadian Continuous Positive Airway Pressure for Patients with Central Sleep Apnea and Heart Failure Trial (CANPAP). Circulation 115:3173-3180

21. Meoli AL, Casey KR, Clark RW, Coleman JA Jr, Fayle RW, Troell RJ, Iber C (2001) Hypopnea in sleep-disordered breathing in adults. Sleep 24:469-470

22. Kuzniar TJ, Kasibowska-Kuzniar K, Freedom T (2012) Trials of bilevel positive airway pressure - spontaneous in patients with complex sleep apnea. Pneumonol Alergol Pol 80:214-219

23. Gay PC (2008) Complex sleep apnea: it really is a disease. J Clin Sleep Med 4:403-405

24. Malhotra A, Bertisch S, Wellman A (2008) Complex sleep apnea: it isn't really a disease. J Clin Sleep Med 4:406-408

25. Kuzniar TJ, Morgenthaler TI (2008) Treatment of complex sleep apnea syndrome. Curr Treat Options Neurol 10:336-341

26. Popescu G, Latham M, Allgar V, Elliott MW (2001) Continuous positive airway pressure for sleep apnoea/hypopnoea syndrome: usefulness of a 2 week trial to identify factors associated with long term use. Thorax 56:727-733

27. Morgenthaler TI, Kuzniar TJ, McLain W, Wolfe L, Fry J, Goldberg R, Rahangdale S (2012) Treatment of complex sleep apnea syndrome with continuous positive airway pressure versus adaptive servoventilation - a prospective randomized study. SLEEP (abstract).

28. Javaheri S, Malik A, Smith J, Chung E (2008) Adaptive pressure support servoventilation: a novel treatment for sleep apnea associated with use of opioids. J Clin Sleep Med 4:305-310

29. Talcott B, Morgenthaler TI, Silber M (2007) Opioid use and sleep disordered breathing: a case control study. Sleep 30(supplement):A160

30. Morgenthaler TI (2008) The quest for stability in an unstable world: adaptive servoventilation in opioid induced complex sleep apnea syndrome. J Clin Sleep Med 4:321-323

31. Salloum A, Rowley JA, Mateika JH, Chowdhuri S, Omran Q, Badr MS (2010) Increased propensity for central apnea in patients with obstructive sleep apnea: effect of nasal continuous positive airway pressure. Am J Respir Crit Care Med 181:189-193

32. Yamauchi M, Tamaki S, Yoshikawa M, Ohnishi Y, Nakano H, Jacono FJ, Loparo KA, Strohl KP, Kimura H (2011) Differences in breathing patterning during wakefulness in patients with mixed apnea-dominant vs obstructive-dominant sleep apnea. Chest 140:54-61

33. Kuzniar TJ, Patel S, Nierodzik CL, Smith LC (2011) Comparison of two servo ventilator devices in the treatment of complex sleep apnea. Sleep Med 12:538-541

34. Kuzniar TJ, Morgenthaler TI (2012) Treatment of complex sleep apnea syndrome. Chest 142:1049-1057

35. Yaegashi H, Fujimoto K, Abe H, Orii K, Eda S, Kubo K (2009) Characteristics of Japanese patients with complex sleep apnea syndrome: a retrospective comparison with obstructive sleep apnea syndrome. Intern Med 48:427-432

36. Endo Y, Suzuki M, Inoue Y, Sato M, Namba K, Hasegawa M, Matsuura M (2008) Prevalence of complex sleep apnea among Japanese patients with sleep apnea syndrome. Tohoku J Exp Med 215:349-354 